\title{
Visualizing Worm Micelle Dynamics and Phase Transitions of a Charged Diblock Copolymer in Water
}

\author{
Yan Geng, ${ }^{\dagger}$ Fariyal Ahmed, ${ }^{\dagger}$ Nishant Bhasin, and Dennis E. Discher* \\ Department of Chemical and Biomolecular Engineering, University of Pennsylvania, \\ Philadelphia, Pennsylvania 19104-6391
}

Received: September 8, 2004

\begin{abstract}
Assemblies of block copolymer amphiphiles are sometimes viewed as glassy, frozen, or static colloids, especially in strongly segregating solutions. Here, we visualize by fluorescence microscopy and AFM the dynamics and transitions of single cylindrical micelles and vesicles composed of a charged diblock copolymer in water. In mapping the salt- and $\mathrm{pH}$-dependent phase diagrams of a near-symmetric diblock of poly(acrylic acid)-polybutadiene, low $\mathrm{pH}$ and high salt $\left(\mathrm{NaCl}, \mathrm{CaCl}_{2}\right)$ neutralize and screen the charged corona sufficiently to foster membrane formation and generate vesicles. Decreased salt and neutral $\mathrm{pH}$ increases intra-coronal repulsion and drives a transition to multi-branched cylinders and highly stable, but fluid and flexible, worm micelles. $\mathrm{Ca}^{2+}$ both stiffens cylinders and stabilizes them relative to spheres. Further increase of intra-coronal repulsion generates spherical micelles by fragmentation and pinch-off at the ends of worms. Both the transition kinetics and phase diagrams indicate divalent cation is about 5-10-fold more effective than monovalent in stabilizing all nonspherical morphologies.
\end{abstract}

\section{Introduction}

Self-assemblies of amphiphilic block copolymers are helping to define a rapidly emerging subclass of colloids. ${ }^{1,2}$ Like small molecule surfactants $(<1 \mathrm{kDa})$, diblock copolymer amphiphiles are capable of assembling into spherical and cylindrical micelles as well as vesicles that define at least three stable morphologies. ${ }^{3}$ Electron microscopy (EM) is especially revealing of the tendency to form these and related structures. ${ }^{4}$ While the high molecular weight of block copolymers and the strong association under a range of solution conditions makes EM imaging possible, such visualization of samples in thin, solid films is static. Interfacial and additional "soft" forces at work in assembly (corona repulsion, screened electrostatics, etc.) as well as particular aspects of dynamics are obscured.

Flexibility in design of copolymer "macro"-surfactants nonetheless offers clear material advantages over conventional or biologically derived surfactants, broadening considerably the range of properties achievable through amphiphilic chemistry. ${ }^{4-6}$ Copolymer vesicles, for example, have thicker and tougher membranes as compared to lipid bilayers, and yet they can still be fluid and deformable. ${ }^{7-9}$ Copolymer vesicles and worm and spherical micelles can also be readily cross-linked, leading to dramatic modifications of mechanochemical properties. ${ }^{10-14}$ These features of diblock copolymers make them potentially useful in many applications, ranging from drug delivery and transport to rheological additives and embedded encapsulators. ${ }^{15}$ These features continue to raise questions, however, about the physical nature of the morphologies and the transitions between them.

The shape and structure of charged diblock copolymer aggregates in solution are governed by an especially delicate balance of forces. Interfacial tension between the core and the bulk solvent tends to orient, confine, and stretch the chains.

\footnotetext{
* Corresponding author. E-mail: discher@seas.upenn.edu.

t Equal contributors to this work.
}

Interactions between the solvated corona blocks are repulsive through sterics and electrostatics but might also prove attractive if entanglements and multivalent ions enter. ${ }^{16-18}$ Perturbations of the balance by many factors thus influence aggregate morphology. ${ }^{19-23}$ The first morphological phase diagram of charged diblock copolymers in dilute solution $(<0.1-10$ wt $\%$ polymer) has been mapped out by Eisenberg and co-workers for "crew-cut" copolymers. Relatively short coronas with large (glassy) cores were made with poly(acrylic acid)-polystyrene (e.g., $\mathrm{PAA}_{52}-\mathrm{PS}_{310}$ ), and these were initially studied with respect to solvent effects. With increasing \%water in dioxane, such diblocks form spherical micelles that transition to coexisting spheres and cylinders, then cylinders alone, cylinders and vesicles, and finally vesicles. Because the hydrophilic segment PAA is a weak acid with a net negative charge when dissolved in water, addition of low concentrations of $\mathrm{HCl}, \mathrm{NaCl}$, or $\mathrm{CaCl}_{2}$ (millimolar/micromolar) also induce "crew-cut" PAA-PS into forming cylinders, vesicles, and even large compound vesicle assemblies. ${ }^{20}$ Nonionic copolymers of poly(ethylene oxide)(1,2)-polybutadiene (PEO-PBD) exhibit similar morphologies, in just water, as recently established by Bates and co-workers with a large series of molecular weights $\left(M_{\mathrm{n}}\right)$ and weight fractions of the hydrophilic PEO block $\left(f_{\mathrm{EO}}\right) \cdot{ }^{24}$ As expected from small surfactant paradigms, decreasing $f_{\mathrm{EO}}$ shifts aggregate structures away from spheres and toward smaller mean curvature cylinders and vesicles. Worm micelles predominate in the more symmetric $f_{\text {EO }}$ range of $\sim 0.50-0.55$, whereas spheres form well above this range and vesicles form well below it. The equilibrium boundaries between predominant microphases are also only weakly dependent on $M_{\mathrm{n}}$; that is, phase boundaries in $f_{\mathrm{EO}}$ shift to lower values for PEO-PBD only by about $5-6 \%$ per 100 EO monomers. This finding together with the low glassiness of PBD (versus PS) and its strongly segregating, hydrophobic nature in water may explain why the PEO blocks need not be 
crew-cut to form worm micelles and vesicles. More direct insight into this issue is provided here by a diblock copolymer of PAAPBD.

Here, we investigate the rich phase behavior and dynamics of a single relatively symmetric poly(acrylic acid)-(1,4)-polybutadiene diblock $\left(\mathrm{PAA}_{75}-\mathrm{PBD}_{103}\right.$ with $\left.f_{\mathrm{AA}} \approx 0.5\right)$. Depending on the concentration of added calcium or sodium salt as well as $\mathrm{pH}$, this PAA-PBD diblock is seen to assemble in water into stable spheres, cylinders, or membranes after overnight hydration of a copolymer film. ${ }^{5}$ We show that this symmetric diblock in aqueous solutions near its $\mathrm{p} K_{\mathrm{a}}$ forms cylinders as if it were a neutral diblock (e.g., PEO-PBD). Organic solvents or dialysis into water are not needed. We use fluorescence microscopy (FM), complemented by atomic force microscopy (AFM), to visualize aggregate dynamics as well as structures and interactions in water. Beyond advantages of using liquid water (rather than frozen or fixed samples), FM is also done in thick chambers and not in $\sim 100 \mathrm{~nm}$ thin, constraining films as required for EM. FM thus provides a more equilibrated measure of macroscopic size and stability in the absence of the squeezing and fragmentation that would seem unavoidable with soft objects in thin films (for EM). AFM is exploited for probing hydrated structures directly, providing submicrometer details. While the morphological phase diagrams here appear to be the first made systematically on any copolymer versus salt and $\mathrm{pH}$, our interests lie as much in morphological complexity and relative salt effects as in assessing transitions in solutions approaching physiological (near neutral $\mathrm{pH}, \sim 0.1 \mathrm{M}$ salt). In particular, worm micelles are emerging as systems of great potential for application in drug or dye delivery because they appear able to flow readily through pores, ${ }^{13}$ whereas spherical micelles have already proven to be extremely useful for therapeutic applications. ${ }^{25}$ Here, we visualize the fluidity and flexibility of $\mathrm{PAA}_{75}-\mathrm{PBD}_{103}$ worm micelles as well as the real-time transitions to spheres under a sudden increase of $\mathrm{pH}$ or decrease in salt. We provide quantitative measures of the relative strength of the effects of monovalent $\mathrm{Na}^{+}$and divalent $\mathrm{Ca}^{2+}$ and discuss alternative strategies for generating spherical micelles in situ with an eye toward application.

\section{Experimental Section}

Materials. $\mathrm{PAA}_{75}-\mathrm{PBD}_{103}\left(M_{\mathrm{n}}=5600-5450 \mathrm{~g} / \mathrm{mol}\right.$, polydispersity $=1.10$ ) was synthesized by anionic living polymerization with sequence addition of butadiene and tert-butylarylate, followed by hydrolysis to its acid form (Polymer Source Inc., Montreal). ${ }^{22}$ The copolymer stock solution was made at $10 \mathrm{mg} /$ $\mathrm{mL}$ in chloroform and stored at $4{ }^{\circ} \mathrm{C}$. Organic solvents (chloroform), glass slides, and cover slips were from Fisher; all other chemicals (flourophore dye $\mathrm{PKH} 26, \mathrm{NaCl}, \mathrm{CaCl}_{2}, \mathrm{HCl}$, $\mathrm{NaOH})$ were from Sigma-Aldrich.

Preparation of $\mathbf{P A A}_{75}-\mathbf{P B D}_{103}$ Assemblies by Film Rehydration. Assemblies of copolymer $\mathrm{PAA}_{75}-\mathrm{PBD}_{103}$ at different conditions ( $\mathrm{pH}$, salt concentration) were prepared by film rehydration. Briefly, $10 \mu \mathrm{L}$ of copolymer stock solution was put into a clean glass vial and a uniform polymer film prepared by drying under nitrogen gas and vacuum for $5-7 \mathrm{~h}$. The film was then hydrated with $1 \mathrm{~mL}$ aqueous solutions of desired salt $\left(\mathrm{NaCl}\right.$ or $\left.\mathrm{CaCl}_{2}\right)$ concentration and $\mathrm{pH}$ (by addition of $\mathrm{HCl}$ or $\mathrm{NaOH}$ ) value at $60{ }^{\circ} \mathrm{C}$ for overnight.

Fluorescence Microscopy of Copolymer Assembly Morphology and Kinetics. A hydrophobic fluorescent dye (PKH 26) was added to the copolymer in rehydrated aqueous solutions to visualize the assemblies in a chamber formed between glass slide and coverslip. $\mathrm{PAA}_{75}-\mathrm{PBD}_{103}$ assemblies were imaged with an Olympus IX71 inverted fluorescence microscope using a $60 \mathrm{X}$ objective and a Cascade CCD camera. A $2 \mu \mathrm{L}$ sample was used in the chamber, and approximately 20 pictures were taken per sample.

For kinetic studies on the worm-to-sphere transitions with basic $\mathrm{pH}$ or depletion of $\mathrm{CaCl}_{2}$ by EDTA, a thick chamber of $\sim 5 \mu \mathrm{m}$ was used instead of the typical $\sim 1-2 \mu \mathrm{m}$. First, $10 \mu \mathrm{L}$ of $\mathrm{pH} \approx 7.4$ buffer or $0.1 \mathrm{M}$ EDTA was added to the glass slide first, and then $1 \mu \mathrm{L}$ of the initial worm sample was added on top of the excess base or EDTA. The transition was recorded at approximately 20 frames per second.

AFM Imaging of the Cylinder and Sphere Morphologies. AFM imaging was preformed on a BioScope atomic force microscope (Digital Instruments, Santa Barbara, CA), equipped with a $120 \mu \mathrm{m} x y$ and $6 \mu \mathrm{m} z$ scanner. Sharpened silicon nitride cantilevers (Microlevers, Park Cantilevers) with a spring constant of $10 \mathrm{pN} / \mathrm{nm}$ were used. Samples of PAA $\mathrm{P}_{75}-\mathrm{PBD}_{103}$ copolymer solutions were deposited on glass slides made cationic by precoating with polylysine. AFM tapping mode imaging was done at scan rates of $1-5 \mathrm{~Hz}$.

\section{Results}

Properties of Giant Worm Micelles of PAA $\mathbf{A}_{75}-$ PBD $_{103}$ in Water. PAA is a weak poly-acid with a reported $\mathrm{p} K_{\mathrm{a}} \approx 5^{26}$ that makes this polymer negative and highly hydrophilic upon dilution in water. PBD has a low $T_{\mathrm{g}}$ and is hydrophobic regardless of salt or $\mathrm{pH}$. Like the neutral diblock PEO-PBD, the charged and symmetric amphiphilic diblock copolymer $\mathrm{PAA}_{75}-\mathrm{PBD}_{103}$ directly self-assembles into worm micelles in pure water $(\mathrm{pH} \approx 7$, no added salt) by film rehydration (Figure $1 \mathrm{a}, \mathrm{b})$. The worm micelles are labeled by hydrophobic fluorescent dye (PKH26) and imaged with fluorescence video microscopy.

The contour of these PAA-PBD worm micelles is wellresolved and stable. An average contour length $\langle L\rangle=18 \mu \mathrm{m}$ is estimated from the distribution of measurable contour lengths $(>1 \mu \mathrm{m})$ (Figure 1c). Such length distributions for worm micelles in water prove relatively constant for at least a month, consistent with the product of a strong hydrophobic interaction, $\chi$, and an extended hydrophobic chain length, $N_{\mathrm{h}}$.

Sequential snapshots (Figure 1b) of a single worm micelle in thermal fluctuation demonstrate the flexibility of these giant micelles even though the worms are confined to a thin, pseudo2D gap of $\sim 1 \mu \mathrm{m}$ between glass coverslips (negative surface charge). Overlays of skeletonized contours show that the distance between worm ends, $R$, fluctuates considerably, allowing an evaluation of the thermal average $\left\langle R^{2}\right\rangle=2 l_{\mathrm{p}}^{2}\left[L / l_{\mathrm{p}}-\right.$ $\left.1+\exp \left(-L / l_{\mathrm{p}}\right)\right]$ in terms of the measured contour length $L$ of the worm and the flexibility of the worm as a persistence length, $l_{\mathrm{p}}$. From analysis of many worm micelles (>100 worms), we determine an average $l_{\mathrm{p}}=4 \mu \mathrm{m}( \pm 2 \mu \mathrm{m})$ that considerably exceeds the optical resolution of the microscope $(\sim 250 \mathrm{~nm})$ and also proves consistent with the well resolved but thermally dynamic contour. Both the persistence length and the diameter $d$ of $\mathrm{PAA}_{75}-\mathrm{PBD}_{103}$ worm micelles $(d \approx 25 \mathrm{~nm}$ as measured by AFM) are close to those of worm micelles made from $\mathrm{PEO}_{80}-(1,2)-\mathrm{PBD}_{125}$, which has essentially the same $M_{\mathrm{n}}$. This implies that the weak negative charge of the PAA corona (at $\mathrm{pH}>\mathrm{p} K_{\mathrm{a}}$ ) does not seem to significantly affect the rigidity of these $\mathrm{PAA}_{75}-\mathrm{PBD}_{103}$ worm micelles. Moreover, the finding that the $1,4-\mathrm{PBD}_{103}$ cores here have a diameter similar to that of the micelles of 1,2-PBD 125 suggests that the cores here are more intertwined and entangled than the latter. ${ }^{27}$ They are nonetheless fluid, as suggested below, and by the fact that the stiffness and 
a.

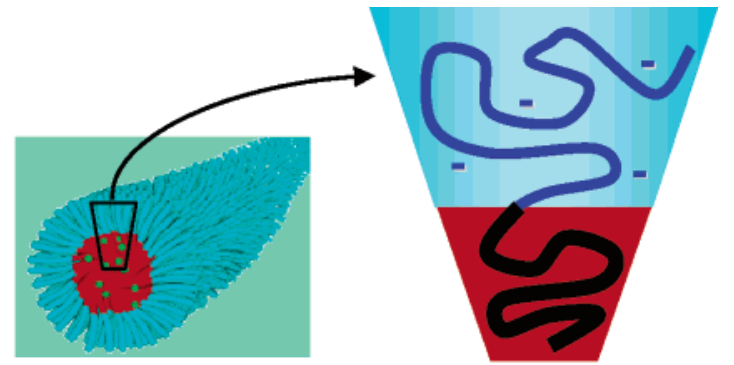

b.
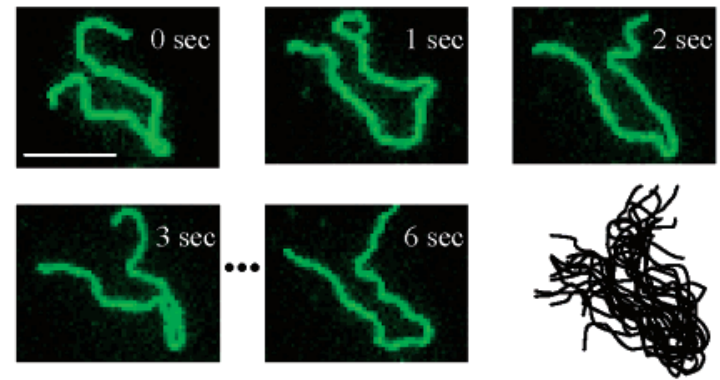

c.

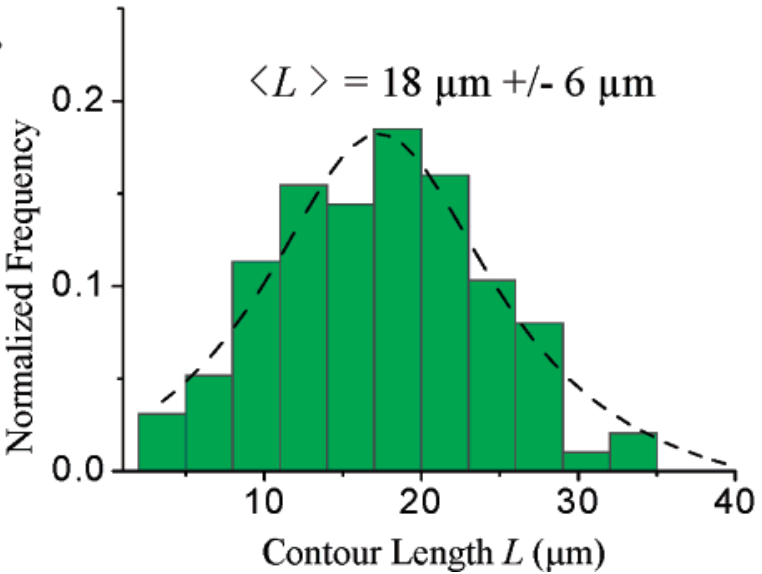

Figure 1. Worm micelles prepared from diblock copolymer $\mathrm{PAA}_{75}$ $\mathrm{PBD}_{103}$ in pure water ( $\mathrm{pH}=7$, no added salt). Worms are fluorescently labeled with a hydrophobic PKH26 dye and confined between two glass coverslips spaced $\sim 1 \mathrm{~mm}$ apart. (a) Schematic illustration of a worm micelle self-assembled from negatively charged diblock. (b) Dynamic snapshots and overlay of 15 backbone traces of a typical $\mathrm{PAA}_{45}-\mathrm{PBD}_{107}$ worm micelle, demonstrating flexibility of these superpolymeric aggregates. Scale bar is $5 \mu \mathrm{m}$. (c) Contour length distribution measured by fluorescence microscopy and fit by a Gaussian (for 200 worms).

diameter of $\mathrm{PAA}_{75}-\mathrm{PBD}_{103}$ worm micelles fit the same $l_{\mathrm{p}} \approx$ $d^{2.8}$ curve as determined for a range of PEO-PBD worm micelles. ${ }^{27}$

Fluidity of the $\mathrm{PAA}_{75}-\mathrm{PBD}_{103}$ worm micelles was studied more directly by fluorescence recovery after photobleaching (FRAP). ${ }^{9}$ A small section of the fluorescently labeled worm micelle was photobleached, $L_{\mathrm{b}}$, and the fluorescent intensity of this section was seen to recover with time by diffusion from the unbleached section of the micelle (Figure 2). The diffusivity of the PKH26 dye within the micelle's core is estimated to be $D \approx 1.3 \mu \mathrm{m}^{2} / \mathrm{s}$. This diffusivity is similar to that of lipids in membranes and probably has more to do with the relatively small size of the probe $(\sim 500 \mathrm{~g} / \mathrm{mol})$. It nonetheless suggests polymeric fluidity rather than a frozen micellar core.

Morphologies of $\mathbf{P A} \mathbf{A}_{\mathbf{7 5}}-\mathbf{P B D}_{\mathbf{1 0 3}}$ in Water. Repulsive interactions between negatively charged PAA segments in the hydrated corona clearly play a significant role in determining the morphology of the $\mathrm{PAA}_{75}-\mathrm{PBD}_{103}$ aggregates in water. Addition of salt $\left(\mathrm{CaCl}_{2}\right.$ or $\left.\mathrm{NaCl}\right)$ that screens repulsions or else addition of an acid to protonate PAA and neutralize its charge a.

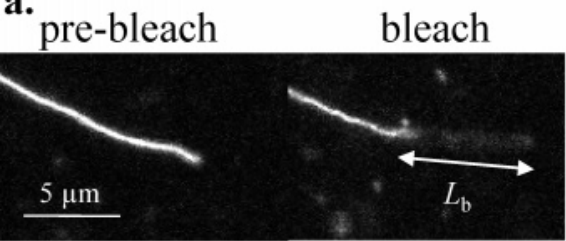

$+25 \mathrm{sec}$

$+45 \mathrm{sec}$
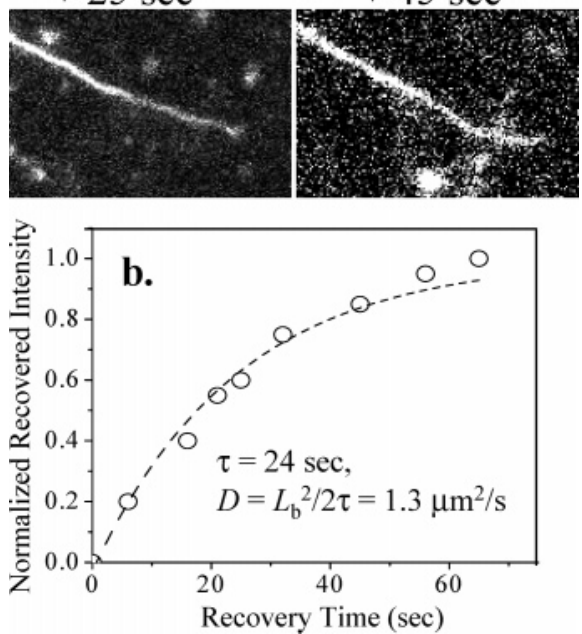

Figure 2. Fluidity of $\mathrm{PAA}_{75}-\mathrm{PBD}_{103}$ worm micelles demonstrated by fluorescence recovery after photobleach (FRAP). (a) Small end portion of a fluorescent worm micelle $\left(L_{\mathrm{b}}\right)$ was photobleached by sustained excitation. Subsequent imagings showed the fluorescent recovery of this bleached portion over time, due to the diffusion of fluorescent PKH dye from the unbleached segment. (b) Recovered intensity within the initially bleached segment was normalized by the intensity of the unbleached segment to account for bleaching during imaging. The recovery time constant $\tau$ was estimated $\tau \approx 24 \mathrm{~s}$ from fitting with the exponential curve: normalized intensity $\approx \exp (t / \tau)$ and $1 \mathrm{D}$ diffusivity was determined by the equation $D=L_{\mathrm{b}}{ }^{2} / 2 \tau=1.3 \mu \mathrm{m}^{2} / \mathrm{s}$.

reduces the repulsion between PAA segments and leads to morphologies of lower curvature. Figure $3 a-c$ illustrates this progressive transition from spherical micelles (S) through various cylindrical micelle (C) morphologies, including worms, and then vesicles (V) and their assembly variants. Note the systematic structural changes from the cylindrical worm micelles (W) to Y-branched (Y) and multi-branched (B) structures with ultimate formation of a large network $(\mathrm{N})$ of cylinders just before pure vesicles form. At the junctions of the various branched micelles, the saddle points tend to reduce net surface curvature by introducing a negative curvature $R^{-1}$ (see Figure $3 \mathrm{~b}$ ) that increasingly approaches the micellar curvature $\approx(d / 2)^{-1}$ as branch density increases. While PEO-PBD copolymers of slightly higher MW appear to show similar branched morphologies that may differ in length scale, ${ }^{24}$ transitions with crewcut PAA-PS copolymer in water plus solvent occur via unbranched cylinders to vesicles. ${ }^{23}$ At extremes in salt and acidity here, and beyond the formation of stable vesicles, a tendency toward inverted phases tends to manifest as higherorder vesicle (HOV) phases with diverse structures (Figure $3 \mathrm{c}$ ).

Morphological Phase Diagrams versus Salt and pH. Phase diagrams were made systematically from $\mathrm{pH} 3$ to 8 and at salt concentrations comparable to the acrylic acid monomer concentration of $0.1 \mathrm{mg} / \mathrm{mL}$ copolymer ([acrylic acid monomer] $\approx 0.68 \mathrm{mM}$ ). $\mathrm{NaCl}$ ranged from $0 \%$ to $200 \%$ of acrylic acid (i.e., up to $1.4 \mathrm{mM}$ ), and $\mathrm{CaCl}_{2}$ ranged from $0 \%$ to $40 \%$ (up to $0.27 \mathrm{mM}$ ). Addition of $\mathrm{HCl}$ or $\mathrm{NaOH}$ was used to set the $\mathrm{pH}$. Buffers were avoided because they contain salt ( $>$ millimolar) as well. Net concentrations of both added salt and acid/base were thus kept less than $\sim$ millimolar, with the intention of 
a.

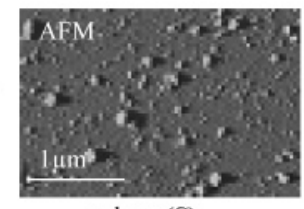

sphere (S)
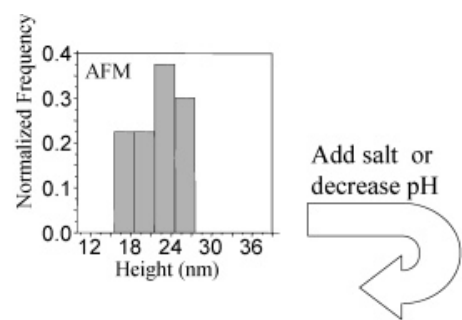

b.

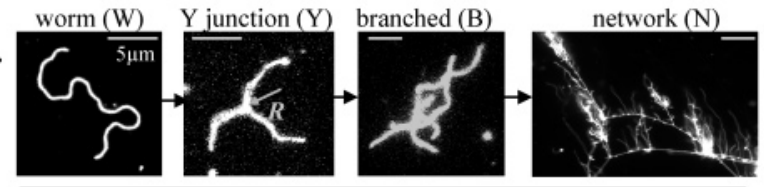

cylinder (C)

c. Vesicle(V) and Higher-Order Vesicle Structures (HOV)

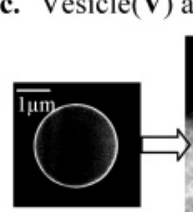

vesicle $(\mathbf{V})$

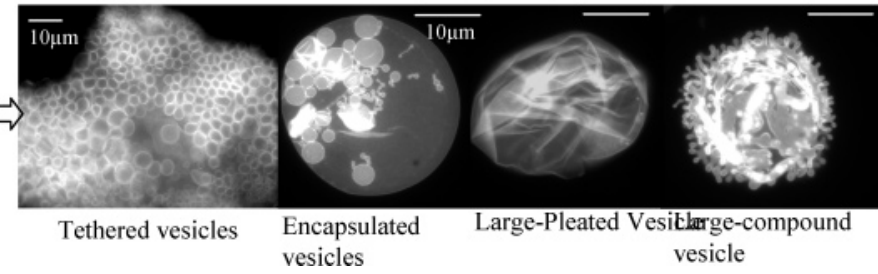

Figure 3. Salt- and $\mathrm{pH}$-dependent $\mathrm{PAA}_{75}-\mathrm{PBD}_{103}$ assemblies. (a) Spherical micelles imaged by AFM. (b) Cylindrical micelles with different structures. (c) Isolated vesicle and various higher-order vesicle (HOV) structures.

influencing only PAA segment interactions without affecting bulk properties of the solution such as ionic strength, osmotic pressure, etc., which might also influence aggregate morphology. ${ }^{28}$ Nearly 100 samples span the various morphologies shown in Figure 3 and were used to obtain the phase diagrams in Figure 4. Smooth lines sketched between different symbols establish the phase boundaries for different morphologies. With increasing salt and acidity, the boundaries establish the classic sequence of self-assembled spheres (S), cylinders (C), and vesicles (V), as separated by broad coexistence regions $(\mathrm{C}+\mathrm{S}, \mathrm{V}+\mathrm{C})$. At low as well as high $\mathrm{pH}$, the two phase diagrams appear to superimpose if rescaled by a factor of $\sim 5$.

Spherical micelles predominate above $\mathrm{pH} \approx 7.4$ and at very low salt (S phase in Figure 4). Tapping mode AFM was used to image the morphology of immobilized micelles, as shown in Figure 3a. Micelles were bound to mica that was precoated with polylysine, which is cationic and thus opposite in charge to PAA over the entire $\mathrm{pH}$ range studied. This cationic precoating proved necessary for reproducible raster-imaging and provides a simple confirmation of PAA's negative charge. Although AFM taps down and compresses micelles (hydrodynamically at least) as part of the raster imaging process, AFM yields an apparent micelle height or diameter that averages 20$30 \mathrm{~nm}$. These results were supported by dynamic light scattering (DLS).

Spherical micelles predominate when repulsions between increasingly negative PAA segments are strong. Nonetheless, giant wormlike micelles are still prominent in fluorescence imaging at a solution $\mathrm{pH}=8$ when divalent salt is $\geq 3 \% \mathrm{CaCl}_{2}$. This minimum at $\mathrm{pH}=8$ increases to $\sim 30 \% \mathrm{NaCl}$ in switching to monovalent cation. The factor of $\sim 10$ difference between transitions with divalent calcium and monovalent sodium is suggestive of an exponential dependence on valency, which is consistent with screening length considerations and perhaps cooperative cross-linking by $\mathrm{Ca}^{2+}$ ion.

Transitions and Stiffness of Cylindrical Micelles with Salt and pH. AFM tapping mode was further used to directly resolve the various cylinder morphologies in the absence of added dye.
The morphological transitions obtained by AFM are consistent with FM. With increasing $\mathrm{pH}$ (no salt), the cylindrical structures clearly shed branches and junctions to become classical worm micelles (Figure 5). At $\mathrm{pH}=3.8$, multi-branched structures are dominant, but at $\mathrm{pH}=5, \mathrm{Y}$-junctions are most common. At $\mathrm{pH}=6$, worm micelles predominate, but spheres also dot the substrate. Note that the flexed configurations for all of the cylinder morphologies are suggestive of meso-scale or longer persistence lengths $(\sim 100-1000 \mathrm{~nm})$. Regardless of the transitions, all cylindrical structures also have about the same diameter in AFM as the spherical micelles in Figure 3a. This implies minimal changes in corona dimensions, which likely restricts transformation pathways.

Within the broad region of coexistence between the various cylinders and spheres but below $\mathrm{pH} \approx 7.0$, cylindrical micelles lose any optically visible junctions (Figure 6, inset). Over the same $\mathrm{pH}$ range, the worms also decrease in length almost linearly with increasing $\mathrm{pH}$ (Figure 6). Average contour lengths of $\sim 40 \mu \mathrm{m}$ decrease to $\sim 20 \mu \mathrm{m}$, with $\mathrm{CaCl}_{2}(\leq 7 \%)$ providing clear stabilization of longer lengths. $\mathrm{NaCl}$ has a similar effect in stabilizing long worms, but $\mathrm{NaCl}$ is again about 10 times less effective than $\mathrm{CaCl}_{2}$. At $\mathrm{pH}=7$, for example, it takes about $60 \% \mathrm{NaCl}$ to get the same worm length as in $7 \% \mathrm{CaCl}_{2}$.

Added $\mathrm{CaCl}_{2}$ significantly increases the stiffness of cylinders. Figure $7 \mathrm{a}$ demonstrates a nonlinear increase in the average worm persistence length with added $\mathrm{CaCl}_{2}$ (at $\mathrm{pH}=7$ ). Snapshots and overlayed traces of a single $22 \mu \mathrm{m}$ worm at $10 \% \mathrm{CaCl}_{2}$ in Figure $7 \mathrm{~b}$ clearly illustrate how much less flexible it is $\left(l_{\mathrm{p}}=\right.$ $13 \mu \mathrm{m})$ than a worm in pure water $\left(l_{\mathrm{p}}=4 \mu \mathrm{m}\right)$ with a similar contour length (Figure $1 \mathrm{~b}$ ). The increase in $l_{\mathrm{p}}$ has the form of a saturable binding isotherm, which seems consistent with divalent $\mathrm{Ca}^{2+}$ associating with the hydrated PAA headgroups. Fitting the data with a simple hyperbola (ignoring the distinction between free and total $\mathrm{CaCl}_{2}$ ) gives a half-max $\left[\mathrm{CaCl}_{2}\right]=4.8 \%$ and a maximum persistence length of $16.8 \mu \mathrm{m}$.

In Figure 6, beyond the decreasing length with $\mathrm{pH}$ up to $\mathrm{pH}$ $\approx 7$, the plot also shows that over a very narrow range of $\mathrm{pH}$ from 7 up to 7.4 (no salt; and up to $\mathrm{pH} 8$ with $7 \% \mathrm{CaCl}_{2}$ ), the 

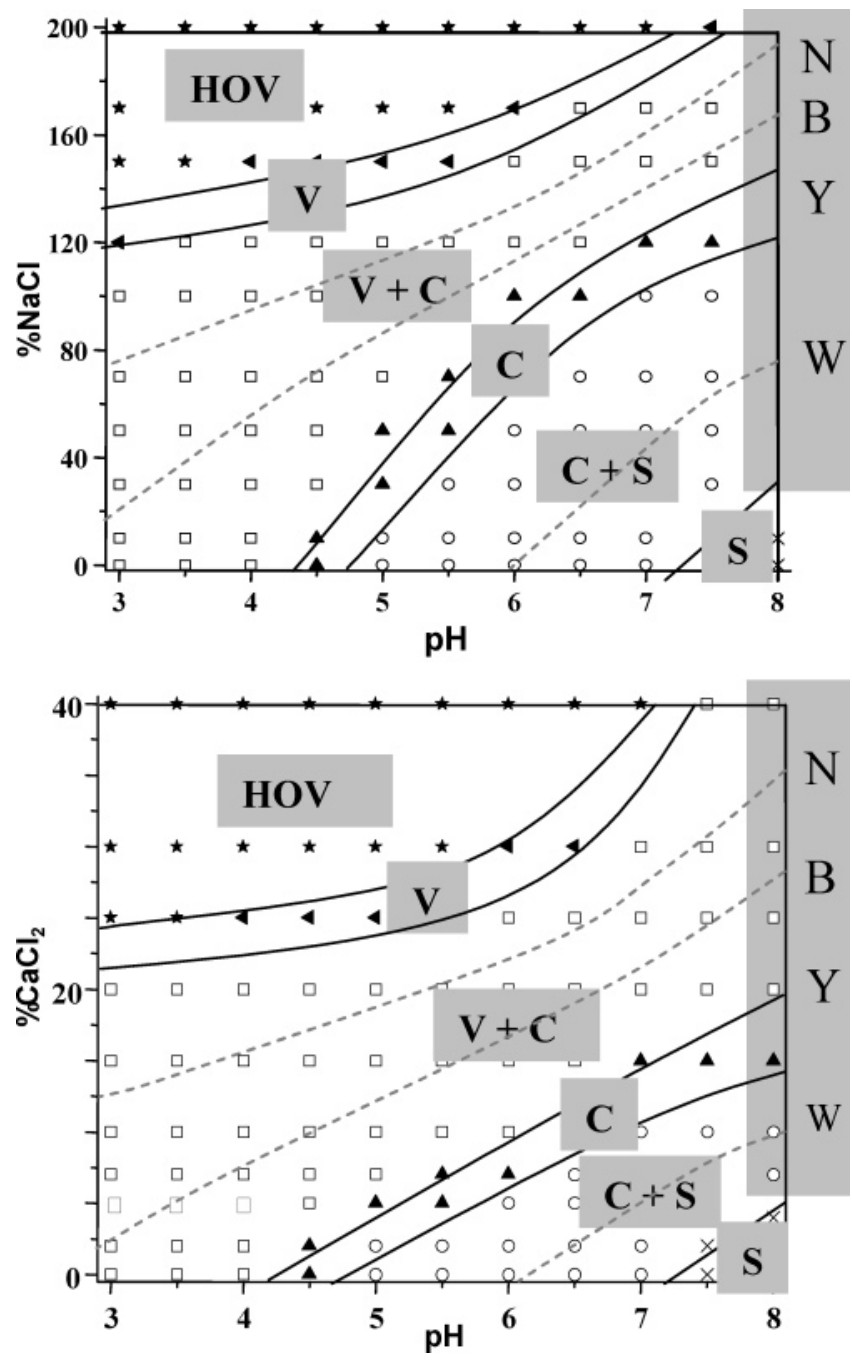

Figure 4. Morphological phase diagrams for $\mathrm{PAA}_{75}-\mathrm{PBD}_{103}$ in water $\left(0.1 \mathrm{mg} / \mathrm{mL}\right.$ polymer) as a function of $\mathrm{pH}$ and added salt $\left(\mathrm{NaCl}, \mathrm{CaCl}_{2}\right)$. The salt concentration is converted to molar percentage of salt concentration relative to total acrylic acid monomer concentration of $\mathrm{PAA}_{75}-\mathrm{PBD}_{107}: \%$ salt $=$ [salt $] /[$ acrylic acid]. Different symbols represent different morphologies: $\times$ sphere, $\bigcirc$ cylinder + sphere, $\Delta$ cylinder, $\square$ vesicle+cylinder, tilted $\boldsymbol{\Delta}$ vesicle, $\star$ higher-order vesicle (HOV). Within the cylindrical region, its structure transits from worm (W), to Y junction (Y), multi-branched (B), and ultimate network formation $(\mathrm{N})$ with added salt and reduced $\mathrm{pH}$.

giant micelles rapidly shrink to lengths essentially unresolvable by fluorescence microscopy (i.e., $<1 \mu \mathrm{m}$ ). This very rapid decay in preferred worm micelle length with a small $\mathrm{pH}$ change that increases corona repulsion is consistent with a precipitous, firstorder transition. The transition is pushed to higher $\mathrm{pH}$ with added $\mathrm{CaCl}_{2}$, which is once again consistent with stabilization of worm micelles versus spheres by divalent cation.
Real-Time Dynamics of Worm-to-Sphere Transition with pH and Salt. Transition pathways are not necessarily clear from phase diagrams. Whether a worm micelle transforms to spheres by fragmenting along its contour or by pinching off spheres only at its ends is not obvious. Linear length decreases noted above might suggest incremental subtractions perhaps at the ends. However, marked decreases above $\mathrm{pH} \approx 7$ suggest more dramatic transformations.

Quickly increasing solution $\mathrm{pH}$ from 4.4 to 7.4 (salt concentration kept constant) shows the real-time dynamics of a wormto-sphere transition (Figure 8a,b). The transition occurs within seconds and indicates that deprotonation of the PAA corona by base rapidly changes the molecular shape of $\mathrm{PAA}_{75}-\mathrm{PBD}_{103}$ in water. A long and flexible worm breaks segmentally into shorter worms (Figure 8a), whereas short worms or rods pinch off from the end and eventually break further into spherical micelles (Figure 8b). This extends kinetic scattering studies of Eisenberg and co-workers on short rods of PAA-PS, which suggest a short rod "end-cap" of higher energy is susceptible to severing into stable spherical micelles. ${ }^{29}$ Likewise, near the ends of worm micelles, Jain and Bates reported a peristaltic morphology prone to pinch-off and akin to a Rayleigh instability. ${ }^{30}$

Quickly decreasing solution $\mathrm{Ca}^{2+}$ (from $12 \%$ ) by adding excess EDTA (pH kept at 7.4) shows, Figure 8c, a slower wormto-sphere transition than the $\mathrm{pH}$ change. The strong chelation of $\mathrm{Ca}^{2+}$ by EDTA is effective but clearly takes time, tens of minutes, to cause the worm to break into spherical micelles. This is clearly much slower than the base-induced transition. Binding of $\mathrm{Ca}^{2+}$ into the PAA corona is evidently much stronger than $\mathrm{H}^{+}$and is thus more difficult to remove.

Plotting the maximum contour lengths of the worms/rods above versus time (Figure 8d) demonstrates that the worm-tosphere transitions follow an exponential decay, $L=L_{\text {Initial }} \times$ $\exp ^{(-t / \tau)}$. For the $\mathrm{pH}$-induced transitions of Figure 8 , both the long worm and the short worm/rod exhibit similar time constants of $\tau \approx 0.5-0.6 \mathrm{~s}$. In contrast, the EDTA chelation of $\mathrm{Ca}^{2+}$ fits with $\tau \approx 140 \mathrm{~s}$.

\section{Discussion}

The charged, symmetric diblock copolymer $\mathrm{PAA}_{75}-\mathrm{PBD}_{103}$ self-assembles in water into a variety of giant, micrometer-size vesicle and cylindrical microphases, exhibiting particularly informative dynamics in the latter morphologies. Visualized here in real-time by fluorescence microscopy (FM), the worm micelles prove stable and fluid, but highly susceptible to changes in $\mathrm{pH}$ and salt. Figure 9a illustrates the underlying energetics of the transitions viewed along a diagonal in the phase diagrams of Figure 4. Divalent calcium clearly stabilizes each preferred morphology and also stiffens at least the cylinder structures; calcium proves 5-10-fold more stabilizing than monovalent sodium. Indeed, the phase diagrams here may be the first
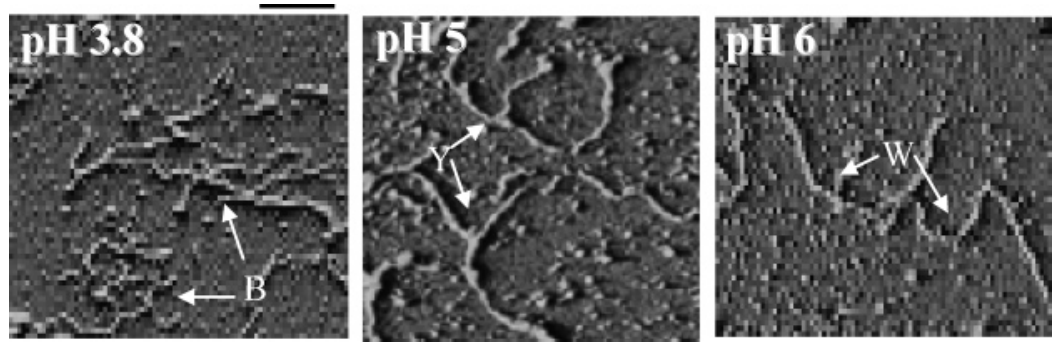

Figure 5. AFM tapping mode images of cylindrical structure changes with increasing $\mathrm{pH}$. Scale bar is $5 \mu \mathrm{m}$. The average height or diameter of the worms is $27 \pm 5 \mathrm{~nm}$ independent of $\mathrm{pH}$. 


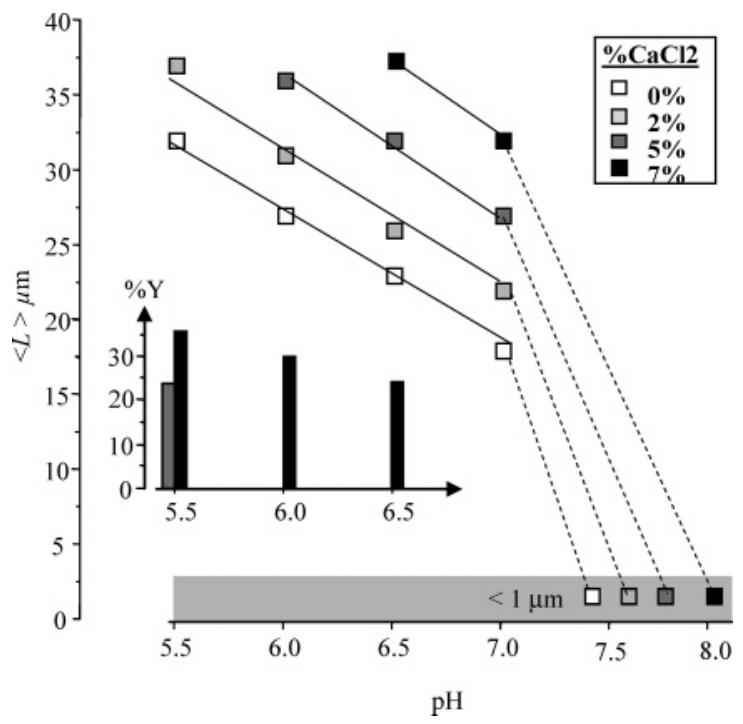

Figure 6. Average worm contour length and \% Y-junctions (inset) within the worm-dominant cylinder phase as functions of $\mathrm{pH}$ and $\mathrm{CaCl}_{2}$. Among all cylindrical micelles $(\mathrm{Y}+\mathrm{W}), \% \mathrm{Y}$ was estimated from its fluorescence intensity fraction: $\% \mathrm{Y}=I_{\mathrm{Y}} /\left(I_{\mathrm{Y}}+I_{\mathrm{W}}\right)$.

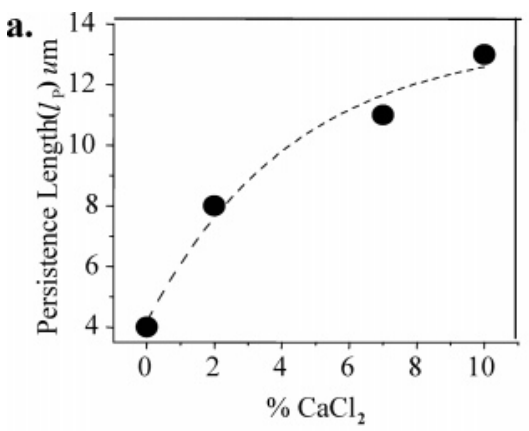

b.

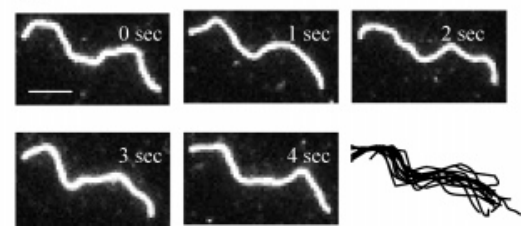

Figure 7. $\mathrm{PAA}_{75}-\mathrm{PBD}_{103}$ worm micelles stiffen with addition of $\mathrm{CaCl}_{2}$. (a) Average persistence length $\left(l_{\mathrm{P}}\right)$ increases with $\% \mathrm{CaCl}_{2}$ added to aqueous solutions at $\mathrm{pH} \approx 7$. (b) Snapshots and overlay of 15 backbone traces of a worm after addition of $10 \% \mathrm{CaCl}_{2}$. Scale bar is $5 \mu \mathrm{m}$.

systematic reports for any charged diblock amphiphile, but the phase diagrams more importantly reinforce a simple rescaling by nearly collapsing on each other when the calcium axis is multiplied by $\sim 5$. Simple ideas of screening from DebyeHuckel theory would suggest a rescaling of approximately $e^{+2 /}$ $e^{+1} \approx 2.7$, and so a higher value of about $5-10$ is suggestive of cooperative stiffening with divalent cation.

Phase diagrams provide crucial information on the morphology formed by an amphiphilic diblock copolymer under a specific set of conditions. Studying the transition dynamics from one structure to another leads to a better understanding of kinetics and mechanism. Dynamic studies are especially interesting for many biological micelle systems ${ }^{31}$ and membrane electroporation, ${ }^{32}$ and are obviously relevant to drug targeting and release. ${ }^{33}$ Past studies of the dynamics of block copolymer in solution are primarily based on turbidity, SANS, differential scanning calorimetry, and rheology measurements, but not direct and real-time imaging. ${ }^{34,35}$ Eisenberg and co-workers used EM to study the sphere-to-rod, rod-to-sphere, and rod-to-vesicle transitions of "crew-cut" PAA-PS with different solvent compositions, by quenching the transition intermediates in liquid nitrogen and then preserving the aggregate morphologies using freeze-drying technique. ${ }^{29,36}$ Here, we captured the real-time worm-to-sphere transitions of $\mathrm{PAA}_{75}-\mathrm{PBD}_{103}$ by $\mathrm{FM}$ with $\mathrm{pH}$ or salt jumps, allowing convenient imaging of the fragmentation and pinch-off process.

Worm micelles are especially interesting for application in drug delivery because they take up hydrophobic compounds (e.g., fluorophores) and also are able to flow readily through pores. ${ }^{13}$ While spherical micelles have already proven to be extremely useful for therapeutic applications, ${ }^{25}$ the results here clearly show that destabilized worms transform to spherical micelles under suitable perturbations. With this in mind, one strategy that we are already pursuing is to generate micelles in situ with degradable amphiphilic block copolymers such as PEO-polycaprolactone (PCL) that start as worm micelles but transform through PCL hydrolysis to increase $f_{\mathrm{EO}}$.

Comparisons with Neutral Copolymer Morphologies. The various morphological "sub"-phases of cylindrical micelles networks, branches, junctions, and worms - are similar to those already mapped for a large series of neutral PEO-PBD (1,2$\mathrm{BD})$ in water. Indeed, symmetric PEO-PBD diblocks tend to yield unbranched worm micelles, and the symmetric (in $f$ ) PAA-PBD diblock used here also yields worm micelles, notably when half-neutralized at $\mathrm{pH} \approx \mathrm{p} K_{\mathrm{a}}$. A distinct phase of networked cylinders ${ }^{24}$ was not observed here; however, highly branched structures coexist with vesicles $(\mathrm{V}+\mathrm{C})$. The driving force for the polymorphism of cylindrical micelles with decreasing $\mathrm{pH}$ and increasing salt is most likely a reduction in local mean curvature. Interestingly, by very different mechanisms, these structural changes emulate biological phenomena such as the $\mathrm{Ca}^{2+}$-fostered dendritic branching of actin filaments with accessory proteins during cell crawling. ${ }^{27}$ Perhaps one day, $\mathrm{Ca}^{2+}$-triggering of cylindrical micelle branching might be harnessed to mimic such biomolecular processes.

Recent studies of PEO-PBD-based worm micelles show the supramolecular persistence length of worm micelles scales with core diameter $d$ as $l_{\mathrm{p}} \approx d^{2.8}$, which is very close to cubic scaling expected of a fluid filament. ${ }^{27}$ With PAA-PBD-based worms, addition of calcium increases $l_{\mathrm{p}}$ from a measured minimum of $4 \mu \mathrm{m}$ to a maximum of $16.8 \mu \mathrm{m}$, with the latter number extrapolated from a saturable binding fit of the stiffening trend (Figure 7). Intercalation of calcium into the corona can be viewed as increasing the effective core diameter $d_{\text {eff }}$ of strong interaction (hydrophobic segregation plus calcium effects). Within a naïve model that calcium binding and/or bridging of PAA segments has an equivalent energy density to the interfacial tension $(\gamma)$ between the core and hydrated corona, the scaling law above $\left(l_{\mathrm{p}} \approx d^{2.8}\right)$ can be used. The worm micelle's $d_{\mathrm{eff}}$ thus increases from $d=d_{\text {core }}$ by about 1.67 -fold upon saturable addition of calcium. Such an increase should be and is less than $\sim 2$-fold, which is the approximate result for a symmetric diblock copolymer: $\left(d_{\text {core }}+d_{\text {corona }}\right) / d_{\text {core }} \approx 2$. The estimation being close to the maximum of two implies, however, that calcium intercalation into the corona can indeed be considered strong and effective.

Vesicle and Higher-Order Vesicle Phase (HOV). The high salt, acid side of the phase diagrams is also of interest, although more for future investigation. As sketched in Figure 9b, the flatter vesicular structures result from neutralization. With PAA this occurs through increased screening as well as increased protonation of corona PAA. A range of studies on PEO-PBD 

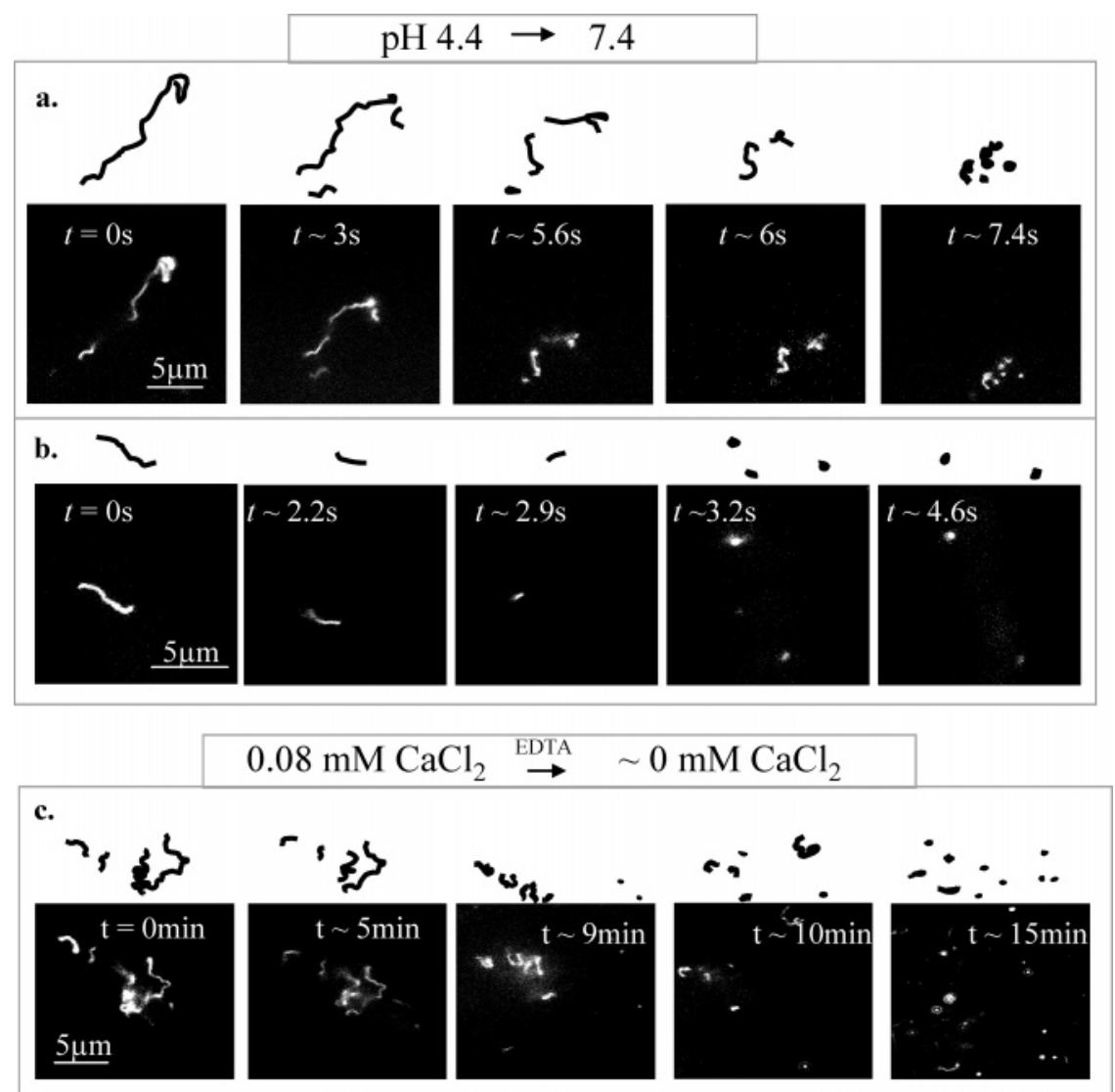

d.

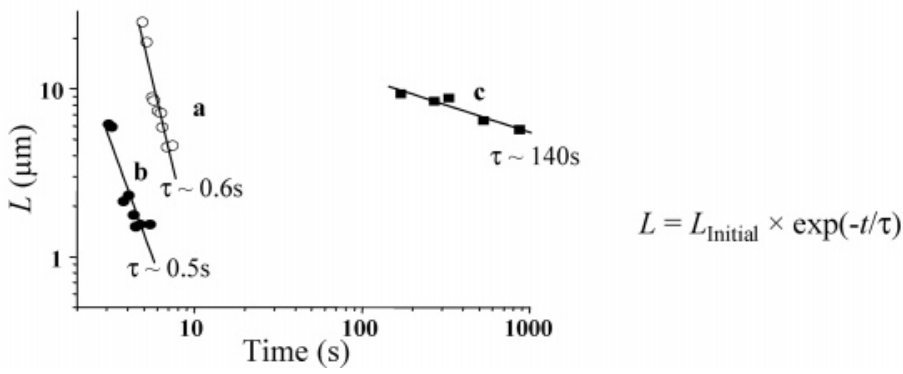

Figure 8. Dynamic worm-to-sphere transitions with increasing $\mathrm{pH}(\mathrm{a}, \mathrm{b})$ or vanishing salt (c). Fragmentation of a long worm (a) and pinch-off from the end of a short worm (b) by changing $\mathrm{pH}$ from 4.4 to 7.4 (salt concentration kept constant). (c) Dynamic worm-to-sphere transition induced by depleting $\mathrm{CaCl}_{2}$ with EDTA ( $\mathrm{pH}$ kept at 7.4). (d) The decreasing maximium worm lengths versus time were fit by exponential decays.

micelles $^{27}$ and vesicles ${ }^{37}$ have also clearly indicated that the flattening between these morphologies occurs as the structure thins and the area per molecule increases. The membranes could also certainly prove more interdigitated.

Higher-order vesicle (HOV, Figure 3c) phases with diverse structures are formed in extreme neutralization beyond the simple vesicle phase in both $\mathrm{NaCl}$ and $\mathrm{CaCl}_{2}$ solutions (Figure 4). The variety of HOV structures include tightly tethered "tissue-like" vesicle aggregate and multi-vesicles encapsulated in one large vesicle, resembling the hierarchical self-assembly of membranes in cells. It seems unlikely that the formation of these self-assembled vesicle structures is strictly due to cationic bridging of PAA segments because monovalent $\mathrm{Na}^{+}$is also capable of driving the HOV formation.

HOVs with large pleats or collapsed and layered membranes, and compound vesicles that resemble aggregated soap bubbles also seen here (Figure 3c), have also been observed by Eisenberg and co-workers with "crew-cut" PAA-PS after dialysis from solvent into water. ${ }^{20}$ The finding that these higher-order vesicle structures are much larger than typical vesicles in the $\mathrm{V}$ regime $(1-5 \mu \mathrm{m}$ in average) certainly suggests a tendency for flatter membranes. They are also stable: these samples of HOV obtained after film rehydration were left at room temperature and periodically checked by FM for 5 months without coalescence. The interconnections between vesicles thus suggest a thermodynamic extrapolation beyond the membrane structure of Figure 9 with locally inverted structures that give rise to hemifused and related stem structures. As with liposomes, the vesicle in vesicle embedding could provide a timing mechanism for designing controlled release block copolymer devices. ${ }^{20,38}$

\section{Conclusions}

With a nonglassy core of PBD and a near-symmetric block of hydrophilic PAA, the $\mathrm{PAA}_{45}-\mathrm{PBD}_{107}$ diblock copolymer reported here directly self-assembles into stable but fluid and flexible micelles and vesicles in water. The vesicles and cylindrical micelles are micrometer-sized structures clearly visualized by fluorescence microscopy, which accesses colloidal dynamics of these structures as soon as they form. Both salts $\left(\mathrm{NaCl}\right.$ or $\left.\mathrm{CaCl}_{2}\right)$ and $\mathrm{pH}$ control aggregate morphology with the classic sequence of self-assembled structures being vesicles that transition to cylinders and cylinders that transition to sphere 


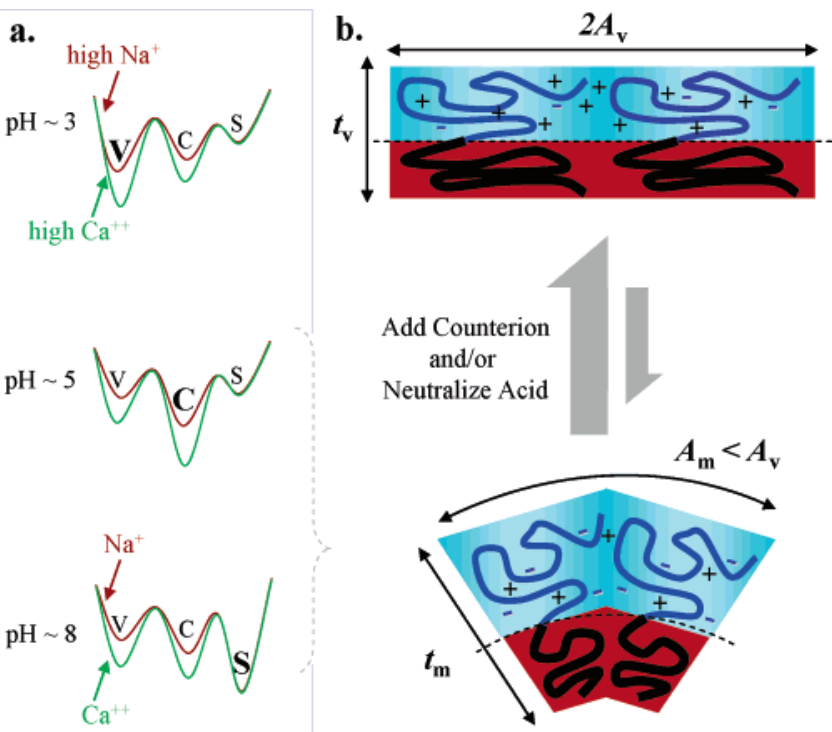

Figure 9. Free energy per molecule (a) and molecular-scale shape changes (b) that underlie morphological transitions with charge changes in the corona. In the latter, $t$ is the thickness of corona plus core and $A$ is the area per copolymer, where subscript $\mathrm{v}$ denotes vesicle and $\mathrm{m}$ denotes micelle.

micelles. Broad coexistence regions are also observed, as others have reported. In addition, divalent $\mathrm{Ca}^{2+}$ stiffens cylinders and is 5-10-fold more effective than monovalent $\mathrm{Na}^{+}$in stabilizing morphologies. Decreasing the intra-corona repulsions of worm micelles drives worm micelles to grow to tens of micrometers in average length before branching and network formation emerge, sequentially, as the mean curvature is reduced. Giant vesicles and higher-order vesicle assemblies eventually form, consistent with curvature minimization. Increasing intra-corona repulsion by decreasing salt and $\mathrm{pH}$ leads to discontinuous shortening of worm micelles and generation of spherical micelles. Visualizing the real-time dynamic worm-to-sphere transition, which occurs within seconds after a $\mathrm{pH}$ step and minutes after $\mathrm{CaCl}_{2}$ chelation by EDTA, demonstrates that long worm micelles are prone to fragment into shorter worms, which then pinch-off from their ends. The results here thus add further insight into the colloidal dynamics of these stable, but morphologically complex, block copolymer aggregates.

Acknowledgment. We thank Prof. Laurence Romsted for useful discussion and careful reading of the manuscript. Funding was provided by Penn's NSF-MRSEC, the Penn-Drexel NanoTech Institute, and an NIH R21 grant.

\section{References and Notes}

(1) Gelbart, W. M.; Ben-Shaul, A. J. Phys. Chem. 1996, 100, 13169.
(2) Evans, D. F.; Wennerstrom, H. The Colloidal Domain: Where Physics, Chemistry, Biology, and Techology Meet, 2nd ed.; VCH: New York, 1998.

(3) Alexandridis, P.; Lindman, B. Amphiphilic Block Copolymers: Selfassembly and Applications; Elsevier: New York, 2000.

(4) Won, Y. Y.; Davis, H. T.; Bates, F. S. Science 1999, 283, 960

(5) Discher, B. M.; Won, Y. Y.; Ede, D. S.; Lee, J. C. M.; Bates, F. S.; Discher, D. E.; Hammer, D. A. Science 1999, 284, 1143.

(6) Pakstis, L.; Nowak, A. P.; Deming, T. J.; Pochan, D. J. Biomacromolecules 2004, 5, 312-318.

(7) Lipowsky, R.; Sackmann, E. Structure and Dynamics of Membranes; Elsevier: New York, 1995.

(8) Bermudez, H.; Aranda-Espinaza, H.; Hammer, D. A.; Discher, D. E. Europhys. Lett. 2003, 64, 4-10.

(9) Lee, J. C. M.; Santore, M.; Bates, F. S.; Discher, D. E. Macromolecules 2002, 35, 323-326.

(10) Discher, B. M.; Bermudez, H.; Hammer, D. A.; Discher, D. E. J. Phys. Chem. B 2002, 106, 2848-2854.

(11) Ahmed, F.; Hategan, A.; Discher, D. E.; Discher, B. M. Langmuir 2003, 19, 6505-6511. 7712 .

12) Nardin, C.; Winterhalter, M.; Meier, W. Langmuir 2000, 16, 7708-

(13) Dalhaimer, P.; Bates, F. S.; Discher, D. E. Macromolecules 2003, $36,6873-6877$.

(14) Ding, J.; Liu, G. Macromolecules 1998, 31, 6554-6558.

(15) Discher, D. E.; Eisenberg, A. Science 2000, 297, 967.

(16) Israelachvili, J. Intermolecular \& Surface Forces; Academic Press: London, 1992.

(17) Helfand, E.; Wasserman, Z. R. Developments in Block Copolymers; Goodman: New York, 1982; Vol. I.

(18) Forster, S.; Abetz, V.; Muller, A. H. E. Adv. Polym. Sci. 2004, $166,173-210$

(19) Zhang, L.; Eisenberg, A. Science 1995, 268, 1728-1731.

(20) Zhang, L.; Yu, K.; Eisenberg, A. Science 1996, 272, 1777-1779.

(21) Shen, H.; Zhang, L.; Eisenberg, A. J. Phys. Chem. B 1997, 101 , 4697-4708.

(22) Yu, K.; Zhang, L.; Eisenberg, A. Langmuir 1996, 12, 5980-5984.

(23) Shen, H.; Eisenberg, A. J. Phys. Chem. B 1999, 103, 9473-9487.

(24) Jain, S.; Bates, F. S. Science 2003, 300, 460-464.

(25) Yokoyama, M.; Okano, T.; Sakurai, Y.; Ekimoto, H.; Shibazaki, C.; Kataoka, K. Cancer Res. 1991, 51, 3229-36.

(26) Chen, K. M.; Jiang, X.; Kimerling, L. C.; Hammond, P. T. Langmuir 2000, 16, 7825-7834.

(27) Dalhaimer, P.; Bermudez, H.; Discher, D. E. J. Polym. Sci., Part B: Polym. Phys. 2003, 42, 168-176.

(28) Rager, T.; Meryer, W. H.; Wegner, G.; Winnik, M. A. Macromolecules 1997, 30, 4911-4919.

(29) Burke, S.; Eisenberg, A. Langmuir 2001, 17, 6705-6714.

(30) Jain, S.; Bates, F. S. Macromolecules 2004, 37, 1511-1523.

(31) Bender, M. Interfacial Phenomena in Biological Systems; Marcel Dekker: New York, 1991.

(32) Egbaria, K.; Weiner, N. N. Adv. Drug Delivery Rev. 1990, 5, 287.

(33) Kakorin, S.; Stoylov, S. P.; Neumann, E. Biophys. Chem. 1996, $58,1$.

(34) Kim, J. K.; Lee, H. R.; Gu, T. C.; Jeong, Y. H. Macromolecules 1998, 31, 4045-4048.

(35) Krishnamoorti, R.; Silva, A. S.; Modi, M.; Hammouda, B. Macromolecules 2000, 33, 3803-3809.

(36) Chen, L.; Shen, H.; Eisenberg, A. J. Phys. Chem. B 1999, 103 9488-9497.

(37) Bermudez, H.; Brannan, A. K.; Hammer, D. A.; Bates, F. S. Macromolecules 2002, 35, 8203-8208.

(38) Chiruvolu, S.; Walker, S.; Israelachvili, J.; Schmitt, F. J.; Leckband, D.; Zasadzinski, J. A. Science 1994, 264, 1753-1756. 